\title{
ON THE CLASS OF FUNCTIONS CONVEX IN THE NEGATIVE DIRECTION OF THE IMAGINARY AXIS
}

\author{
ADAM LECKO \\ (Received 17 July 2000; revised 14 February 2001)
}

Communicated by P. C. Fenton

\begin{abstract}
In this paper we present a new proof of the equivalence of the analytic and the geometric characterization of the class of functions convex in the negative or positive direction of the imaginary axis.

2000 Mathematics subject classification: primary $30 \mathrm{C} 45$.

Keywords and phrases: functions convex in the negative direction of the imaginary axis, geometric characterization, Julia Lemma.
\end{abstract}

\section{Introduction}

Let $\overline{\mathbb{C}}=\mathbb{C} \cup\{\infty\}$, let $\mathbb{D}=\{z \in \mathbb{C}:|z|<1\}$ denote the open disk in the plane, and let $\mathbb{T}=\partial \mathbb{D}$. For each $k>0$, let

$$
\mathbb{O}_{k}=\left\{z \in \mathbb{D}: \frac{|1-z|^{2}}{1-|z|^{2}}<k\right\}
$$

denote the disk in $\mathbb{D}$ called an oricycle, such that the boundary circle $\partial \mathbb{O}_{k}$ is tangent at $z=1$ to the unit circle $\mathbb{T}$. The Julia Lemma ([4]; see also [1, page 56]) which is recalled below is the basis for our considerations.

LeMma 1.1 (Julia). Let $\omega$ be an analytic function in $\mathbb{D}$ with $|\omega(z)|<1$, for $z \in \mathbb{D}$. Assume that there exists a sequence $\left(z_{n}\right), n \in \mathbb{N}$, of points in $\mathbb{D}$ such that $\lim _{n \rightarrow \infty} z_{n}=1$, $\lim _{n \rightarrow \infty} \omega\left(z_{n}\right)=1$ and

$$
\lim _{n \rightarrow \infty} \frac{1-\left|\omega\left(z_{n}\right)\right|}{1-\left|z_{n}\right|}=\alpha<\infty .
$$

(C) 2002 Australian Mathematical Society $1446-7887 / 2000 \$ A 2.00+0.00$ 
Then

$$
\frac{|1-\omega(z)|^{2}}{1-|\omega(z)|^{2}} \leq \alpha \frac{|1-z|^{2}}{1-|z|^{2}}, \quad z \in \mathbb{D},
$$

and hence, for every $k>0, \omega\left(\mathbb{O}_{k}\right) \subset \mathbb{O}_{\alpha k}$.

REMARK 1.1. Since

$$
\frac{1-|\omega(z)|}{1-|z|} \geq \frac{1-|\omega(0)|}{1+|\omega(0)|}, \quad z \in \mathbb{D},
$$

for every function $\omega$ analytic in $\mathbb{D}$ with $|\omega(z)|<1$ for $z \in \mathbb{D}$, the constant $\alpha$ defined in (1.1) is positive (see [1, page 43]).

\section{Convexity in the negative direction of the imaginary axis}

2.1. For $w \in \mathbb{C}$ and $\theta \in[0,2 \pi)$, let $l[w, \theta]=\left\{w+t e^{i \theta}: t \in[0,+\infty)\right\}$. For $A, B \subset \mathbb{C}$ and $w \in \mathbb{C}$, let

$$
A \pm B=\{u \pm v \in \mathbb{C}: u \in A \wedge v \in B\}, \quad A+w=A+\{w\} .
$$

2.2. Let us start with the following definition.

DEFINITION 2.1. A domain $\Omega \subset \mathbb{C}, \Omega \neq \mathbb{C}$, is called convex in the negative direction of the imaginary axis if and only if the half-line $l[w, 3 \pi / 2]$ is contained in $\mathbb{C} \backslash \Omega$ for every $w \in \mathbb{C} \backslash \Omega$. The set of all such domains will be denoted by $\mathscr{Z}^{-}$.

Obviously, $\Omega \in \mathscr{Z}^{-}$if and only if the half-line $l[w, \pi / 2]$ is contained in $\Omega$ for every $w \in \Omega$.

DEFINITION 2.2. Let $\mathscr{C} \mathscr{V}^{-}$denote the class of all analytic and univalent functions $f$ in $\mathbb{D}$ such that $f(\mathbb{D})$ is in $\mathscr{Z}^{-}$. Functions in the class $\mathscr{C} \mathscr{V}^{-}$will be called convex in the negative direction of the imaginary axis.

2.3. Now we introduce, for an arbitrary domain in $\mathscr{Z}^{-}$, a special selected nullchain $\left(C_{n}\right)$.

\section{Construction of a prime end for the domain convex in the negative direction of the imaginary axis}

Let us recall that a crosscut $C$ of a domain $G \subset \overline{\mathbb{C}}$ is an open Jordan arc in $G$ such that $\bar{C}=C \cup\{a, b\}$, where $a, b \in \partial G$. Let $\Omega \in \mathscr{Z}^{-}$be an arbitrary domain. 
1. Assume first that $\Omega$ is neither a vertical strip nor a half-plane with the boundary straight line parallel to the imaginary axis. Then there exists $w_{0} \in \partial \Omega$ such that the vertical half-line $l\left[w_{0}, \pi / 2\right] \backslash\left\{w_{0}\right\}$ starting from $w_{0}$ is contained in $\Omega$. For each $t \in(0, \infty)$, let us denote $C(t)=\left\{w \in \mathbb{C}:\left|w-w_{0}\right|=t\right\}$. It is clear that $\Omega \cap C(t) \neq \emptyset$ for every $t \in(0, \infty)$. By Proposition 2.13 in [6, page 28], for each $t \in(0, \infty)$ there are countably many crosscuts $C_{k}(t) \subset C(t), k \in \mathbb{N}$, of $\Omega$ each of which is an arc of the circle $C(t)$. By $\Omega_{0}(t) \subset \Omega$ we denote the component of $\Omega \backslash C(t)$ containing the half-line $l\left[w_{0}+i t, \pi / 2\right] \backslash\left\{w_{0}+i t\right\}$ and by $Q(t) \in \bigcup_{k \in N} C_{k}(t)$ we denote the crosscut containing the point $w_{0}+i t$. So $Q(t) \subset \partial \Omega_{0}(t)$. Let now $\left(t_{n}\right), n \in \mathbb{N}$, be a strictly increasing sequence of points in $(0, \infty)$ such that $\lim _{n \rightarrow \infty} t_{n}=\infty$ and let $\left(Q\left(t_{n}\right)\right)$ be the corresponding sequence of crosscuts of $\Omega$. It is easy to observe that

(1) $\overline{Q\left(t_{n}\right)} \cap \overline{Q\left(t_{n+1}\right)}=\emptyset$ for every $n \in \mathbb{N}$.

(2) $\Omega_{0}\left(t_{n+1}\right) \subset \Omega_{0}\left(t_{n}\right)$ for every $n \in \mathbb{N}$.

(3) $\operatorname{diam}^{*} Q\left(t_{n}\right) \rightarrow 0$ as $n \rightarrow \infty$, where $\operatorname{diam}^{*} B$ means the spherical diameter of the set $B \subset \mathbb{C}$.

Therefore $\left(C_{n}\right)=\left(Q\left(t_{n}\right)\right)$ forms a null chain of $\Omega$ (see [6, page 29]). Notice also that the null chain $\left(C_{n}\right)$ is independent of the choice of the sequence $\left(t_{n}\right)$.

The equivalence class of the null chain $\left(C_{n}\right)$ defines the prime end denoted by $p_{\infty}(\Omega)$. We can also show that infinity is a unique principal point of the prime end $p_{\infty}(\Omega)$.

2. (a) Let $\Omega$ be a vertical strip of width $d>0$. Let $w_{0} \in \partial \Omega$ be an arbitrary point. For each $t \in(d, \infty)$, set $C(t)=\left\{w \in \mathbb{C}:\left|w-w_{0}\right|=t\right\}$. It is clear that $\Omega \cap C(t) \neq \emptyset$ for every $t \in(d, \infty)$. Observe that $\Omega(t)$ is a sum of two disjoint circular arcs, denoted by $Q^{+}(t)$ and $Q^{-}(t)$. Let $Q^{+}(t)$ be the circular arc which lies above $Q^{-}(t)$. Precisely, $Q^{+}(t)$ cuts the boundary straight lines of $\Omega$ at two points $w_{1}(t)$ and $w_{2}(t)$, and together with two half-lines $l\left[w_{1}(t), \pi / 2\right]$ and $l\left[w_{2}(t), \pi / 2\right]$ is a boundary of a domain denoted by $\Omega^{+}(t)$. Moreover, $\Omega^{+}(t) \subset \Omega$ and $\Omega^{+}(t) \cap \operatorname{Int} C(t)=\emptyset$.

Let now $\left(t_{n}\right), n \in \mathbb{N}$, be a strictly increasing sequence of points in $(d, \infty)$ such that $\lim _{n \rightarrow \infty} t_{n}=\infty$, and let $\left(Q^{+}\left(t_{n}\right)\right)$ be the corresponding sequence of crosscuts of $\Omega$. It is easy to observe that the conditions (1)-(3) listed in Part 1 of this construction are fulfilled. Therefore $\left(C_{n}^{+}\right)=\left(Q^{+}\left(t_{n}\right)\right)$ forms a null chain of $\Omega$. The null chain $\left(C_{n}^{+}\right)$is independent of the choice of the sequence $\left(t_{n}\right)$.

The equivalence class of the null chain $\left(C_{n}^{+}\right)$defines the prime end denoted by $p_{\infty}^{+}(\Omega)$. We can also say that infinity is a unique principal point of the prime end $p_{\infty}^{+}(\Omega)$

In a similar way the sequence $\left(Q^{-}\left(t_{n}\right)\right)$ is a null chain which represents the second prime end $p_{\infty}^{-}(\Omega)$, different than $p_{\infty}^{+}(\Omega)$.

For the next considerations, the prime end $p_{\infty}^{+}(\Omega)$ will be denoted by $p_{\infty}(\Omega)$.

(b) Let now $\Omega$ be a half-plane with the boundary straight line parallel to the imaginary axis. Let $w_{0} \in \partial \Omega$ be an arbitrary point. For each $t \in(0, \infty)$, let 
$C(t)=\left\{w \in \mathbb{C}:\left|w-w_{0}\right|=t\right\}$. It is clear that $Q(t)=\Omega \cap C(t)$ is a half-circle for every $t>0$. Repeating considerations similar to those above we see that the sequence $\left(C_{n}\right)=\left(Q\left(t_{n}\right)\right)$, for an arbitrary strictly increasing sequence $\left(t_{n}\right), n \in \mathbb{N}$, of points in $(0, \infty)$ such that $\lim _{n \rightarrow \infty} t_{n}=\infty$, forms a null chain of $\Omega$ which represents a prime end denoted by $p_{\infty}(\Omega)$.

In this way we construct for every domain $\Omega$ in $\mathscr{Z}^{-}$, in a unique way, a prime end $p_{\infty}(\Omega)$.

Let $f$ be a conformal mapping $\mathbb{D}$ onto $\Omega$, this is, let $f \in \mathscr{C} \mathscr{V}^{-}$. By the prime end theorem there exists a bijective mapping $\widehat{f}$ of the unit circle $\mathbb{T}$ onto the set of all prime ends of $\Omega\left(\left[6\right.\right.$, page 30]). Hence there is a unique $\zeta_{\infty} \in \mathbb{T}$ such that $p_{\infty}(\Omega)=\widehat{f}\left(\zeta_{\infty}\right)$. We can also show that infinity is a unique principal point of the prime end $p_{\infty}(\Omega)$.

\section{An analytic characterization of the class of function convex in the negative direction of the imaginary axis}

3.1. In the proof of the main theorem, which analytically characterizes the class $\mathscr{C} \mathscr{V}^{-}$, we will need the following lemma.

LEMMA 3.1. Let $\left(a_{n}\right), n \in \mathbb{N}$, be a sequence such that $a_{n}>0, n \in \mathbb{N}$, and

$$
\lim _{n \rightarrow \infty}\left(a_{1} a_{2} \cdots a_{n}\right)=0 .
$$

Then there exists a convergent subsequence $\left(a_{n_{k}}\right), k \in \mathbb{N}$, of the sequence $\left(a_{n}\right)$. Moreover $0 \leq \lim _{k \rightarrow \infty} a_{n_{k}}=a \leq 1$.

ProOF. Suppose that only finitely many elements of the sequence $\left(a_{n}\right)$ lie in the interval $(0,1]$. Then $a_{n}>1$ for sufficiently large $n$, which contradicts (3.1). This means that infinitely many elements of the sequence $\left(a_{n}\right)$ lie in the interval $(0,1]$. Taking a convergent subsequence $\left(a_{n_{k}}\right), k \in \mathbb{N}$, of the sequence $\left(a_{n}\right)$ completes the proof.

3.2. Now we will prove the theorem which says that every function $f$ in the class $\mathscr{C} \mathscr{V}^{-}$, with $p_{\infty}(f(\mathbb{D}))=\widehat{f}(1)$, preserves convexity in the negative direction of the imaginary axis on every oricycle $\mathbb{O}_{k}$.

THEOREM 3.1. Let $f$ be an analytic and univalent function in $\mathbb{D}$. Then $f \in \mathscr{C} \mathscr{V}^{-}$ and $p_{\infty}(f(\mathbb{D}))=\widehat{f}(1)$, if and only if $f\left(\mathbb{O}_{k}\right) \in \mathscr{Z}^{-}$for every $k>0$.

PRoOF. 1. Assume that $f \in \mathscr{C} \mathscr{V}^{-}$and $\zeta_{\infty}=1$ corresponds to the prime end $p_{\infty}(f(\mathbb{D}))$. For each $t \in(0, \infty)$, let us define the function

$$
\omega_{t}(z)=f^{-1}(f(z)+i t), \quad z \in \mathbb{D} .
$$


Since $f(\mathbb{D})$ is a domain convex in the negative direction of the imaginary axis, $f(z)+i t \in f(\mathbb{D})$ for every $t \in(0, \infty)$ and $z \in \mathbb{D}$. Hence, from the univalence of $f$, it follows that the function $\omega_{t}$ is well defined for each $t \in(0, \infty)$.

For every domain $\Omega \in \mathscr{Z}^{-}$, we select two points $w_{0} \in \partial \Omega$ and $w_{1} \in \Omega$, in the following way. If $\Omega$ is not a vertical strip or a half-plane with the boundary straight line parallel to the imaginary axis, then there exists $w_{0}$ in $\partial \Omega$ such that the half-line $l\left[w_{0}, \pi / 2\right] \backslash\left\{w_{0}\right\}$ lies in $\Omega$. Let $w_{1} \in \Omega$ be an arbitrary point lying on this half-line.

In the case when $\Omega$ is a vertical strip or a half-plane with the boundary straight line parallel to the imaginary axis, let $w_{1} \in \Omega$ be an arbitrary point and $w_{0} \in \partial \Omega$ be such that $\operatorname{Im} w_{1}=\operatorname{Im} w_{0}$.

Assume now that for the domain $f(\mathbb{D})$ the points $w_{0}$ and $w_{1}$ are chosen as above. Of course $l\left[w_{1}, \pi / 2\right]$ lies in $f(\mathbb{D})$. Let us fix $t \in(0, \infty)$ and let us consider the sequence $\left(w_{n}\right)=\left(w_{1}+i t_{n}\right)$ of points in $l\left[w_{1}, \pi / 2\right]$ and the corresponding sequence $\left(z_{n}\right)=\left(f^{-1}\left(w_{n}\right)\right)$ of points in $\mathbb{D}$, where $t_{n}=(n-1) t, n \in \mathbb{N}$.

With the same notation as in the construction of a prime end for the domain in the class $\mathscr{Z}^{-}$, let $C\left(t_{n}\right)=\left\{w \in \mathbb{C}:\left|w-w_{0}\right|=\left|w_{n}-w_{0}\right|\right\}$ and let $Q\left(t_{n}\right) \subset C\left(t_{n}\right)$, for $n \in \mathbb{N}$, denote the crosscut of $f(\mathbb{D})$ containing the point $w_{n}$. From the method of choosing $w_{0}$ and $w_{1}$ we see that the conditions (1)-(3) are satisfied and $\left(Q\left(t_{n}\right)\right)$ is a null-chain representing the prime end $p_{\infty}(f(\mathbb{D}))$. By the prime end theorem $\left(f^{-1}\left(Q\left(t_{n}\right)\right)\right)$ is a null-chain in $\mathbb{D}$ that separates the origin from $\zeta_{\infty}=1$ for large $n$. Since $z_{n}=f^{-1}\left(w_{n}\right) \in f^{-1}\left(Q\left(t_{n}\right)\right)$ and $\operatorname{diam} f^{-1}\left(Q\left(t_{n}\right)\right) \rightarrow 0$ for $n \rightarrow \infty$, we conclude that $\lim _{n \rightarrow \infty} z_{n}=1$. Observe that $\omega_{t}\left(z_{n}\right)=f^{-1}\left(w_{n}+i t\right)=z_{n+1}$. Let now

$$
a_{n}=\frac{1-\left|\omega_{t}\left(z_{n}\right)\right|}{1-\left|z_{n}\right|}, \quad n \in \mathbb{N} \text {. }
$$

Hence

$$
a_{n}=\frac{1-\left|\omega_{t}\left(z_{n}\right)\right|}{1-\left|z_{n}\right|}=\frac{1-\left|z_{n+1}\right|}{1-\left|z_{n}\right|}
$$

for all $n \in \mathbb{N}$. Consequently,

$$
\begin{aligned}
\lim _{n \rightarrow \infty}\left(a_{1} a_{2} \cdots a_{n}\right) & =\lim _{n \rightarrow \infty}\left(\frac{1-\left|z_{2}\right|}{1-\left|z_{1}\right|} \frac{1-\left|z_{3}\right|}{1-\left|z_{2}\right|} \cdots \frac{1-\left|z_{n}\right|}{1-\left|z_{n-1}\right|} \frac{1-\left|z_{n+1}\right|}{1-\left|z_{n}\right|}\right) \\
& =\lim _{n \rightarrow \infty} \frac{1-\left|z_{n+1}\right|}{1-\left|z_{1}\right|}=0 .
\end{aligned}
$$

By Lemma 3.1 there exists a convergent subsequence $\left(a_{n_{k}}\right), k \in \mathbb{N}$, of the sequence $\left(a_{n}\right)$ such that $0 \leq \lim _{k \rightarrow \infty} a_{n_{k}}=\alpha(t) \leq 1$. Hence we conclude that there exists a convergent subsequence $\left(z_{n_{k}}\right)$ of the sequence $\left(z_{n}\right)$ such that

$$
\lim _{k \rightarrow \infty} \frac{1-\left|\omega_{t}\left(z_{n_{k}}\right)\right|}{1-\left|z_{n_{k}}\right|}=\alpha(t) \leq 1
$$


for every fixed $t \in(0, \infty)$. In fact, in view of Remark 1.1, $\alpha(t)>0$ for every $t \in(0, \infty)$.

In this way, for each $t \in(0, \infty)$, the function $\omega_{t}$ satisfies the assumptions of the Julia Lemma. Hence, and by the fact that $\alpha(t) \leq 1$ for every $t \in(0, \infty)$, we have

$$
\omega_{t}\left(\mathbb{O}_{k}\right) \subset \mathbb{O}_{\alpha(t) k} \subset \mathbb{O}_{k}
$$

for every $k>0$.

Fixing now $k>0$ we see from (3.2) that $f^{-1}\left(f\left(\mathbb{O}_{k}\right)+i t\right) \subset \mathbb{O}_{k}$, so $f\left(\mathbb{O}_{k}\right)+i t \subset$ $f\left(\mathbb{O}_{k}\right)$ for every $t \in(0, \infty)$. Therefore, $f\left(\mathbb{O}_{k}\right) \in \mathscr{Z}$ - for every $k>0$.

2. Let us now assume that $f\left(\mathbb{O}_{k}\right) \in \mathscr{Z}$ - for every $k>0$. Since $\infty \in \partial f\left(\mathbb{O}_{k}\right)$ for every $k>0$ and $f(\mathbb{D})=\bigcup_{k>0} f\left(\mathbb{O}_{k}\right), \infty \in \partial f(\mathbb{D})$ and $f(\mathbb{D})$ is convex in the negative direction of the imaginary axis. Observe also that there exists a prime end $p_{\infty}(f(\mathbb{D}))$ which corresponds to some point $\zeta_{\infty} \in \mathbb{T}$. We need to show that $\zeta_{\infty}=1$. To this end, let $k>0$ be fixed and suppose that $\zeta_{\infty} \neq 1$. Let $\left(Q\left(t_{n}\right)\right), n \in \mathbb{N}$, be an arbitrary sequence of crosscuts of $f(\mathbb{D})$ which represents the prime end $p_{\infty}(f(\mathbb{D}))$ corresponding in a unique way to a point $\zeta_{\infty} \in \mathbb{T}$, that is, $\left(Q\left(t_{n}\right)\right)$ is a null-chain of $f(\mathbb{D})$. By the prime end theorem $\left(f^{-1}\left(Q\left(t_{n}\right)\right)\right)$ is a null-chain that separate in $\mathbb{D}$ the points 0 from $\zeta_{\infty}$ for large $n$. Since $\zeta_{\infty} \neq 1$ and $\operatorname{diam} f^{-1}\left(Q\left(t_{n}\right)\right) \rightarrow 0$ for $n \rightarrow \infty$ we see that

$$
f^{-1}\left(Q\left(t_{n}\right)\right) \cap \mathbb{O}_{k}=\emptyset
$$

for large $n$.

On the other hand, $f\left(\mathbb{O}_{k}\right)$ is in $\mathscr{Z}^{-}$, which implies that $Q\left(t_{n}\right) \cap f\left(\mathbb{O}_{k}\right) \neq \emptyset$ for large $n \in \mathbb{N}$. This contradicts (3.3) and shows that $\zeta_{\infty}=1$ and $p_{\infty}(f(\mathbb{D}))=\widehat{f}(1)$. The proof of the theorem is finished.

Using Theorem 3.1 we are able to find an analytic characterization of functions in the class $\mathscr{C} \mathscr{V}^{-}$.

THEOREM 3.2. If $f \in \mathscr{C} \mathscr{V}^{-}$and $p_{\infty}(f(\mathbb{D}))=\widehat{f}(1)$, then

$$
\operatorname{Im}\left\{(1-z)^{2} f^{\prime}(z)\right\} \geq 0, \quad z \in \mathbb{D} .
$$

PROOF. Let $f \in \mathscr{C} \mathscr{V}^{-}$and $p_{\infty}(f(\mathbb{D}))=\widehat{f(1)}$. By Theorem 3.1 the domain $f\left(\mathbb{O}_{k}\right)$ for every $k>0$, is in the class $\mathscr{Z}^{-}$. This means geometrically that the function

$$
\gamma_{k} \ni z \rightarrow \operatorname{Re} f(z)
$$

is monotonic on the analytic arc $\gamma_{k}=\partial \mathbb{O}_{k} \backslash\{1\}$ for every $k>0$. We will use the following parametrization of $\gamma_{k}$

$$
\gamma_{k}: z=z(\theta)=\frac{1+k e^{i \theta}}{1+k}, \quad \theta \in(0,2 \pi)
$$


Hence in place of (3.5) we consider the function

$$
(0,2 \pi) \ni \theta \rightarrow \operatorname{Re} f(z(\theta)) .
$$

We have

$$
\begin{aligned}
(1-z(\theta))^{2} & =\frac{k^{2}}{(1+k)^{2}}\left(1-e^{i \theta}\right)^{2}=-\frac{4 k \sin ^{2}(\theta / 2)}{(k+1) i}\left(\frac{k}{k+1} e^{i \theta} i\right) \\
& =\frac{4 k \sin ^{2}(\theta / 2)}{k+1} z^{\prime}(\theta) i=2 \operatorname{Re}\{1-z(\theta)\} z^{\prime}(\theta) i, \quad \theta \in(0,2 \pi) .
\end{aligned}
$$

In view of the fact that the arc $\gamma_{k}$ is positively oriented, the same is true of the arc $f\left(\gamma_{k}\right)$, since $f$ is a conformal mapping. Hence

$$
\begin{aligned}
\frac{d}{d \theta} \operatorname{Re} f(z(\theta)) & =\operatorname{Re}\left\{z^{\prime}(\theta) f^{\prime}(z(\theta))\right\} \\
& =\frac{k+1}{4 k \sin ^{2}(\theta / 2)} \operatorname{Re}\left\{-i(1-z(\theta))^{2} f^{\prime}(z(\theta))\right\} \\
& =\frac{1}{2 \operatorname{Re}\{1-z(\theta)\}} \operatorname{Im}\left\{(1-z(\theta))^{2} f^{\prime}(z(\theta))\right\} \geq 0
\end{aligned}
$$

for $\theta \in(0,2 \pi)$, so (3.4) holds.

Now we will prove the converse theorem.

THEOREM 3.3. If $f$ is an analytic function in $\mathbb{D}$ and

$$
\operatorname{Im}\left\{(1-z)^{2} f^{\prime}(z)\right\} \geq 0, \quad z \in \mathbb{D},
$$

then $f \in \mathscr{C} \mathscr{V}^{-}$and $p_{\infty}(f(\mathbb{D}))=\widehat{f}(1)$.

ProOF. Let $f$ be analytic in $\mathbb{D}$ and satisfy (3.9).

1. If there exists a point $z_{0} \in \mathbb{D}$ such that the equality in (3.9) holds, then by the maximum principle for harmonic functions the equality in (3.9) holds in the whole disk $\mathbb{D}$. This implies that there exists a real number $a \in \mathbb{R} \backslash\{0\}$ so that $(1-z)^{2} f^{\prime}(z) \equiv a, z \in \mathbb{D}$. This is satisfied only for the function

$$
f(z)=f_{0}(z)=b+\frac{a}{1-z}, \quad z \in \mathbb{D},
$$

where $b \in \mathbb{C}$. In this case we conclude at once that $f_{0}(\mathbb{D})$ is a half-plane with a straight line as the boundary parallel to the imaginary axis. Hence $f_{0} \in \mathscr{C} \mathscr{V}^{-}$and, as is immediately apparent, $p_{\infty}\left(f_{0}(\mathbb{D})\right)=\widehat{f}_{0}(1)$. Observe also that in this case the function defined in (3.7) is constant on every arc $\gamma_{k}, k>0$, that is, every disk $\mathbb{O}_{k}$ 
is mapped onto the half-plane with a boundary straight line parallel to the imaginary axis.

2. Assume now that in (3.9) strong inequality holds. Since $f$ satisfying (3.9) is close-to-convex with respect to the convex function

$$
h(z)=\frac{-i z}{1-z}, \quad z \in \mathbb{D},
$$

$f$ is univalent in $\mathbb{D}([5])$.

Let us now consider again the function (3.7) defined on the analytic arcs $\gamma_{k}$ : $\partial \mathbb{O}_{k} \backslash\{1\}$ for each $k>0$, parametrized by (3.6). Repeating again the calculations (3.8) we see that the condition (3.9) implies

$$
\frac{d}{d \theta} \operatorname{Re} f(z(\theta))>0, \quad \theta \in(0,2 \pi) .
$$

This means that every positively oriented arc $\gamma_{k}$ which is mapped by the function $f$ satisfying (3.9) onto the positively oriented arc $f\left(\gamma_{k}\right), k>0$, is the boundary of the domain $f\left(\mathbb{O}_{k}\right)$ convex in the direction of the negative imaginary half-axis. By Theorem 3.1, $f \in \mathscr{C} \mathscr{V}^{-}$and $p_{\infty}(f(\mathbb{D}))=\widehat{f}(1)$ which completes the proof of the theorem.

The following theorems are immediate consequences of Theorem 3.2 and Theorem 3.3 by applying them to the function $f(z)=g\left(e^{-i \mu} z\right), z \in \mathbb{D}$, where $g \in \mathscr{C V}^{-}$ and $p_{\infty}(g(\mathbb{D}))=\widehat{g}(1)$.

THEOREM 3.4. If $f \in \mathscr{C} \mathscr{V}^{-}$and $p_{\infty}(f(\mathbb{D}))=\widehat{f}\left(e^{i \mu}\right), \mu \in \mathbb{R}$, then

$$
\operatorname{Im}\left\{e^{i \mu}\left(1-e^{-i \mu} z\right)^{2} f^{\prime}(z)\right\} \geq 0, \quad z \in \mathbb{D} .
$$

THEOREM 3.5. If $f$ is an analytic function in $\mathbb{D}$ and (3.10) is true for $\mu \in \mathbb{R}$, then $f \in \mathscr{C} \mathscr{V}^{-}$and $p_{\infty}(f(\mathbb{D}))=\widehat{f}\left(e^{i \mu}\right)$.

\section{Convexity in the positive direction of the imaginary axis}

The results presented in Section 3 can be applied at once to the functions called convex in the positive direction of the imaginary axis.

DEFINITION 4.1. A domain $\Omega \subset \mathbb{C}, \Omega \neq \mathbb{C}$, will be called convex in the positive direction of the imaginary axis if and only if the half-line $l[w, \pi / 2]$ is contained in $\mathbb{C} \backslash \Omega$ for every $w \in \mathbb{C} \backslash \Omega$ or equivalently if the half-line $l[w, 3 \pi / 2]$ is contained in $\Omega$ for every $w \in \Omega$. The set of all such domains will be denoted by $\mathscr{Z}^{+}$. 
DEFINITION 4.2. Let $\mathscr{C} \mathscr{V}^{+}$denote the class of all analytic and univalent functions $f$ in $\mathbb{D}$ such that $f(\mathbb{D})$ is in $\mathscr{Z}^{+}$. Functions in the class $\mathscr{C} \mathscr{V}^{+}$will be called convex in the positive direction of the imaginary axis.

We can repeat exactly the construction as in Section 2 and find the prime end $p_{\infty}(\Omega)$ for every $\Omega \in \mathscr{Z}^{+}$.

Finally, in view of at Theorem 3.1, Theorem 3.4 and Theorem 3.5 we can formulate the following theorems.

THEOREM 4.1. Let $f$ be an analytic and univalent function in $\mathbb{D}$. Then $f \in \mathscr{C} \mathscr{V}^{+}$ and $p_{\infty}(f(\mathbb{D}))=\widehat{f}(1)$, if and only if $f\left(\mathbb{O}_{k}\right) \in \mathscr{Z}^{+}$for every $k>0$.

THEOREM 4.2. If $f \in \mathscr{C} \mathscr{V}^{+}$and $p_{\infty}(f(\mathbb{D}))=\widehat{f}\left(e^{i \mu}\right), \mu \in \mathbb{R}$, then

$$
\operatorname{Im}\left\{e^{i \mu}\left(1-e^{-i \mu} z\right)^{2} f^{\prime}(z)\right\} \leq 0, \quad z \in \mathbb{D} .
$$

THEOREM 4.3. If $f$ is an analytic function in $\mathbb{D}$ and (4.1) is true for $\mu \in \mathbb{R}$, then $f \in \mathscr{C} \mathscr{V}^{+}$and $p_{\infty}(f(\mathbb{D}))=\widehat{f}\left(e^{i \mu}\right)$.

\section{Remarks}

The class of functions convex in the direction of the imaginary axis, denoted by $\mathscr{C} \mathscr{V}$, was introduced by Robertson [7]. A function $f$, analytic and univalent in $\mathbb{D}$, belongs to $\mathscr{C} \mathscr{V}$ if and only if the domain $f(\mathbb{D})$ is convex in the direction of the imaginary axis, that is, $\left[w_{1}, w_{2}\right] \subset f(\mathbb{D})$ for every $w_{1}$ and $w_{2}$ in $f(\mathbb{D})$ such that $\operatorname{Re} w_{1}=\operatorname{Re} w_{2}$. Robertson proposed an analytic condition to characterize the class $\mathscr{C} \mathscr{V}$ and proved it under some additional assumptions on functions in $\mathscr{C} \mathscr{V}$ connected with the regularity on the unit circle. In the papers [3] and [8] it was shown that the Robertson condition is correct for the whole class $\mathscr{C} \mathscr{V}$.

In fact, the classes $\mathscr{C} \mathscr{V}^{+}$and $\mathscr{C} \mathscr{V}^{-}$are the subclasses of the class $\mathscr{C} \mathscr{V}$ distinguished by Hengartner and Schober [3], where also the analytic conditions (3.4) and (4.1) with $\mu=0$ were demonstrated.

We use the name convex in the negative or positive direction of the imaginary axis following Ciozda [2], where she studied the so-called class $L_{0}$ of functions convex in the direction of the negative real half-axis. To be precise, a function $f$ analytic and univalent in $\mathbb{D}$ is convex in the direction of the negative real half-axis if and only if for every $w \in f(\mathbb{D})$ the half-line $l[w, 0]$ is contained in $f(\mathbb{D})$.

\section{References}

[1] C. Carathéodory, Conformal representation (Cambridge University Press, Cambridge, 1963). 
[2] W. Ciozda, 'Sur la classe des fonctions convexes vers l'axe réel négatif', Bull. Acad. Polon. Sci. 27 (1979), 225-261.

[3] W. Hengartner and G. Schober, 'On schlicht mappings to domains convex in one direction', Comment. Math. Helv. 45 (1970), 303-314.

[4] G. Julia, 'Extension nouvelle d'un lemme de Schwarz', Acta Math. 42 (1918), 349-355.

[5] W. Kaplan, 'Close-to-convex schlicht functions', Mich. Math. J. 1 (1952), 169-185.

[6] Ch. Pommerenke, Boundary behaviour of conformal maps (Springer, Berlin, 1992).

[7] M. S. Robertson, 'Analytic functions star-like in one direction', Amer. J. Math. 58 (1936), 465-472.

[8] W. Royster and M. Ziegler, 'Univalent functions convex in one direction', Publ. Math. Debrecen 23 (1976), 339-345.

Department of Mathematics

Technical University of Rzeszów

ul. W. Pola 2, 35-959 Rzeszów

Poland

e-mail: alecko@prz.rzeszow.pl 